\title{
No apparent transmission of transgenic a-synuclein into nigrostriatal dopaminergic neurons in multiple mouse models
}

\author{
Namratha Sastry ${ }^{1,3}$, Wang Zheng ${ }^{1}$, Guoxiang Liu' ${ }^{1}$, Helen Wang ${ }^{1,4}$, Xi Chen ${ }^{1}$, Michael Cai ${ }^{2,5}$, Parth Contractor ${ }^{1,6}$,
} Carmelo Sgobio', Lixin Sun ${ }^{1}$, Chengsong Xie ${ }^{1}$ and Huaibin Cai ${ }^{1 *}$

\begin{abstract}
Background: a-synuclein (a-syn) is the main component of intracytoplasmic inclusions deposited in the brains of patients with Parkinson's disease (PD) and certain other neurodegenerative disorders. Recent studies have explored the ability of a-syn to propagate between or across neighboring neurons and supposedly "infect" them with a prion-like mechanism. However, much of this research has used stereotaxic injections of heterologous a-syn fibrils to induce the spreading of inclusions in the rodent brains. Whether a-syn is able to transmit from the host cells to their neighboring cells in vivo is unclear.

Methods: Using immunestaining, we examined the potential propagation of a-syn into nigrostriatal dopaminergic (DA) neurons in three lines of transgenic mice that overexpress human wild-type a-syn (ha-syn) in different neuron populations.

Results: After testing for three different routes by which ha-syn propagation might occur, we were unable to find any evidence that ha-syn behaved like a prion and could be transmitted overtime into the DA neurons initially lack of ha-syn expression.

Conclusions: In transgenic mice ha-syn does not have the ability to propagate at pathologically significant levels between or across neurons. It must be noted that these observations do not disprove the studies that show its prion-like qualities, but rather that propagation is not detectable in transgenic models that do not use any injections of heterologous proteins or viral vectors to induce a spreading state.
\end{abstract}

Keywords: Parkinson's disease, a-synuclein, Propagation, Dopaminergic neurons, Transgenic mice

\section{Background}

Parkinson's disease (PD) is the second most common neurodegenerative disease, causing debilitating motor and non-motor symptoms $[1,2]$. PD is pathologically characterized by the death of nigrostriatal dopaminergic neurons in the ventral substantia nigra pars compacta (SNc) of the midbrain, as well as the presence of intracytoplasmic inclusions known as Lewy bodies (LBs) and Lewy neurites (LNs). The main component of these inclusions is $\alpha$-synuclein ( $\alpha-$ syn) [3]. $\alpha-$ syn is a small 140

\footnotetext{
* Correspondence: caih@mail.nih.gov

'Transgenics Section, Laboratory of Neurogenetics, National Institute on Aging, National Institutes of Health, Building 35, Room 1A112, MSC 3707, 35 Convent Drive, Bethesda, MD 20892-3707, USA

Full list of author information is available at the end of the article
}

amino acid protein that is thought to play a role in synaptic vesicle release [4]. Both missense and multiplication mutations of $\alpha$-syn are linked to early onset familial forms of PD [5]. How mutant $\alpha$-syn leads to SNc DA neuron loss and $\mathrm{LB} / \mathrm{LN}$ formation has been under intense investigation ever since.

PD patient brains seem to show a stereotypical appearance of LB/LN pathology that can be mapped into various stages of disease evolution: lesions first appear in the glossopharyngeal and vagal nerves, continue to the SNc DA neurons, and eventually cover the primary sensory and motor cortices [6]. Subsequent studies have hypothesized that $\alpha$-syn intercellular propagation may be responsible for this stereotypical pathology and have provided evidence both in vitro and in vivo [7-10]. The 
in vivo studies have primarily focused on using intracerebral inoculations of diseased brain homogenate or preformed $\alpha$-syn fibrils to study development and progression of PD pathology [9, 10]. In wild-type control mice, injections of preformed fibrils of $\alpha-s y n$ are sufficient to initiate $\mathrm{LB} / \mathrm{LN}$ pathology in regions anatomically connected to the site of injection [9]. Such intracerebral injections can also accelerate the formation of LBs and LNs in otherwise asymptotic mice [10] and can be seen over serial passages of inoculations [11]. This has been further confirmed by a study where inoculation with homogenate from either A53T human $\alpha$-syn transgenic mouse brains or multiple system atrophy patient brains resulted in disease pathology in mice that do not otherwise develop any spontaneous illness [12]. Moreover, this phenomenon has been studied in primates, and propagation is evident in macaque monkeys in addition to rodents [13]. Increasing evidence thus suggests that $\alpha$-syn potentially behaves in a prion-like manner, where mutated $\alpha$-syn can be transmitted from cell to cell and spread the pathology [14].

While these studies provide very compelling results to show the prion-like qualities of $\alpha$-syn, much of the methodology involves artificial injections and inoculations. This triggered us to determine if $\alpha$-syn propagation could be observed in mice that overexpress human wild-type $\alpha$-syn (h $\alpha$-syn), without the need for any injections. Therefore, we generated multiple lines of transgenic mice that overexpress h $\alpha$-syn in different neuron populations inside and outside of the SNc. We then examined three different routes by which $\alpha-s y n$ may propagate into the SNc DA neurons, including long-range propagation from anatomically separated regions, short-distance transmission from presynaptic spiny projection neurons (SPNs), and neighboring DA neurons. Unlike previous inoculation experiments, we found no evidence that $\alpha$-syn could propagate and possess prion-like qualities in any of our three modes of study, thus questioning $\alpha$-syn propagation as the method of disease progression in PD.

\section{Methods}

\section{Ethics statement}

This study was carried out in strict accordance with the recommendations in the Guide for the Care and Use of Laboratory Animals of the National Institutes of Health. The protocol was approved by the Institutional Animal Care and Use Committees of the National Institute of Child Health and Human Development, NIH (Permit Number: 13-040). All surgery was performed under ketamine anesthesia, and all efforts were made to minimize suffering.

\section{Animals}

To generate tetO-SNCA transgenic mice, human wildtype $\alpha$-syn $(S N C A)$ cDNA coding region was inserted into the mouse prion protein ( $\mathrm{pPrP})$-tetO gene expression vector (a gift from Dr. David Borchelt, University of Florida, Gainesville, FL), which is controlled by the tetracycline-responsive promoter (tetP) [15]. The tetOSNCA expression construct was then purified and microinjected into fertilized oocytes derived from C57BL/6 J mice. The founder mice were crossed with wild-type C57BL/6 J mice to produce the F1 generation. Pitx3-tTA knock-in mice were created as described previously [16]. Drd1a-rtTA mice were obtained from Jackson Laboratories (Bar Harbor, ME). All mice were housed in a $12 \mathrm{~h}$ light/dark cycle and fed regular diet ad libitum. All mouse work follows the guidelines approved by the Institutional Animal Care and Use Committees of the National Institute of Child Health and Human Development, NIH.

\section{Genotyping}

Genomic DNA was prepared from tail biopsy using DirectPCR Lysis Reagent (Viagen Biotech, Inc., Los Angeles, CA) and subjected to PCR amplification using specific sets of PCR primers for each genotype, including Pitx3- $t$ TA transgenic mice (Pitx3-F: GACTGGCTTG CCCTCGTCCCA and Pitx3-R: GTGCACCGAGGCCCCAGATCA), tetO-SNCA transgenic mice (PrpEx2-F: TACTGCTCCATTTTGCGTGA and SNCA-R: TCCAG AATTCCTTCCTGTGG), Drd1a-rtTA transgenic mice (14915-F: ACCGGAAGTGCTTTCCTTCT and 14916-R: CGACTTGATGCTCTTGATCTTCC).

\section{Immunohistochemistry and light microscopy}

Mice were sacrificed and then perfused via cardiac infusion with $4 \%$ paraformaldehyde in cold PBS, followed by post-fixation in the same solution overnight. To obtain sections, brain tissues were removed and submerged in $30 \%$ sucrose for $24 \mathrm{~h}$ and sectioned at $30 \mu \mathrm{m}$ thickness using a cryostat (Leica CM1950, Buffalo Grove, IL). Antibodies specific to TH (rabbit polyclonal, 1:1000, Pel-Freez, Rogers, AR), human $\alpha-$ synuclein (mouse monoclonal, syn211, 1:500, Santa Cruz Biotech, Santa Cruz, CA) were used as suggested by manufacturers. Alexa 488 and Alexa 546-conjugated secondary antibodies (1:500, Life Technologies, Grand Island, NY) were used to visualize the staining. Fluorescence images were captured using a laser scanning confocal microscope (LSM 510; Zeiss, Thornwood, NJ). The images were presented as either a single optic layer after acquisition in $\mathrm{z}$-series stack scans at $2-3 \mu \mathrm{m}$ intervals from individual fields or displayed as maximum intensity projections to represent confocal stacks. 


\section{Image analysis}

For the quantitative co-localization assessments, images from serial sections were taken and exported to ImageJ (NIH, Bethesda, MD) for imaging analyses. Each image was split into individual channels for SNCA (488 nm) and $\mathrm{TH}(546 \mathrm{~nm})$. Cell bodies positive for $\mathrm{TH}$ were first selected using the polygon selection tool and then subjected to measurement by mean optical intensities. The mean intensities were then compared to the identical regions in SNCA channel. SNCA intensities below a set threshold were counted as being negative. The overall percentages of positive SNCA cells were then compared between the ages of $1 \mathrm{~m}$ and 16-18 $\mathrm{m}$.

\section{Stereology}

According to the mouse brain in stereotaxic coordinates (3rd edition, Keith B.J. Franklin and George Paxinos), a series of coronal sections across the midbrain $(30 \mu \mathrm{m}$ per section, every fourth section from Bregma $-2.54 \mathrm{~mm}$ to $-4.24 \mathrm{~mm}$ ) were chosen and processed for $\mathrm{TH}$ staining as described above and visualized using a widefield microscope (Axio Imager A1; Zeiss). We examined 16 sections per brain at 40x magnification. The number of $\mathrm{TH}$ positive neurons was assessed using the Optical Fractionator Workflow in Stereo Investigator 11 (MicroBrightField Inc, Williston, VT). The sampling scheme was designed to have coefficient of error (CE) less than $10 \%$ in order to get reliable results. A pilot counting of samples was performed to achieve a total marking of $>200$ cells, which generally yields $\mathrm{CE}<10 \%$. Once the pilot cells counting had completed, the CE was calculated. The counting parameters would be adjusted based on the CE value. To achieve CE $<10 \%$, normally 12 series sections with total 100 counting frames and on average 2 cells per frame would be counted. The final parameters for these studies were: grid size $150 \times 120 \mu \mathrm{m}$ and frame size $50 \times 50 \mu \mathrm{m}$. Three or more mice were used per genotype at each time point.

\section{Tissue fractionation and Western blot}

Striatum tissues were homogenized with 10 volumes of sucrose buffer (0.32 M sucrose, $1 \mathrm{mM}$ NaHCO3, $1 \mathrm{mM}$ $\mathrm{MgCl} 2$, and $0.5 \mathrm{mM} \mathrm{CaCl}$, plus protease and phosphatase inhibitor cocktails) and centrifuged at 10,000 $\mathrm{g}$ for $10 \mathrm{~min}$. Protein concentrations in supernatant were measured by BCA (Thermo Fisher Scientific). Proteins were size-fractioned by 4-12 \% NuPage BisTrispolyacrylamide gel electrophoresis (Life Technologies) using MES running buffer (Life Technologies). After transfer to nitrocellulose membranes, the membranes were immunoblotted with the appropriate dilutions of the primary antibody: human $\alpha-\operatorname{syn}$ (syn211, 1:1000; Santa Cruz Biotechnology, Santa Cruz, CA) and $\alpha$-tubulin (DM1A, 1:2000; Santa Cruz Biotechnology, Santa Cruz,
CA. Signals were visualized with fluorescent secondary antibodies and quantified with ImageJ.

\section{Statistical analysis}

Statistical analysis was performed using Graphpad Prism 5 (Graphpad Software Inc. La Jolla, CA). Data were presented as mean \pm SEM. Statistical significances were determined by comparing means of different groups and conditions using unpaired Student t-test or one-way ANOVA with post hoc Tukey test.

\section{Results}

\section{Transgenic ha-syn is unable to undergo long-range} propagation

We generated a new line of human wild-type $\alpha$-syn $(S N C A)$ transgenic mice under the control of tetracycline operator (tetO): tetO-SNCA. The expression of transgenic $S N C A$ assumes to be regulated by the tetracycline transactivator ( $t T A)$ in a "tet-off" gene expression system [17]. However, immunostaining revealed substantial expression of h $\alpha$-syn in multiple brain regions, including the hippocampus, cerebellum, and cortex, independent of tTA (Fig. 1a). Our first test for propagation utilized the "leaky" expression of h $\alpha$-syn present in tetO-SNCA transgenic mice. We wanted to see whether the tyrosine hydroxylase $(\mathrm{TH})$-positive SNc DA neurons that were initially devoid of h $\alpha$-syn (Fig. 1a) would show any h $\alpha-$ syn accumulation with age. Potentially, these other tissues or residual h $\alpha$-syn from the cerebrospinal fluid can aid in "infecting" the SNc DA neurons. If propagation can occur in this long-range fashion, h $\alpha$-syn might accumulate in SNc DA neurons at advanced ages. We checked for this expression at 1 and 18 months of age (Fig. 1b). At 1-month-old, we found a few ho-syn-positive puncta distributed in the $\mathrm{SNc}$ region; some were spotted inside of SNc DA neurons (Fig. 1b, inset). Any additional h $\alpha$-syn present at later ages would have been evidence for propagation. However, at 18 months of age, we were unable to see any apparent propagation (Fig. 1b). The lack of any substantial accumulation of h $\alpha$-syn in the SNc DA neurons indicates that no long-range propagation is evident for tetO-SNCA mice.

Since the transcription factor paired-like homeodomain 3 (Pitx3) is only expressed by subpopulations of midbrain DA neurons [18], previously we inserted tetracycline transactivator $(t T A)$ coding sequence into the 3 -untranslated region of mouse Pitx3 gene to generate Pitx3-tTA knock-in mice, allowing $t T A$ selectively expressed in midbrain DA neurons [16]. In this so-called "tet-off" system, tTA can turn on the expression of any transgene under the control of tetracycline operator (tetO) [16]. In the absence of such a transgene, this line of mice has no transgenic expression in the midbrain (Fig. 1a, b). 

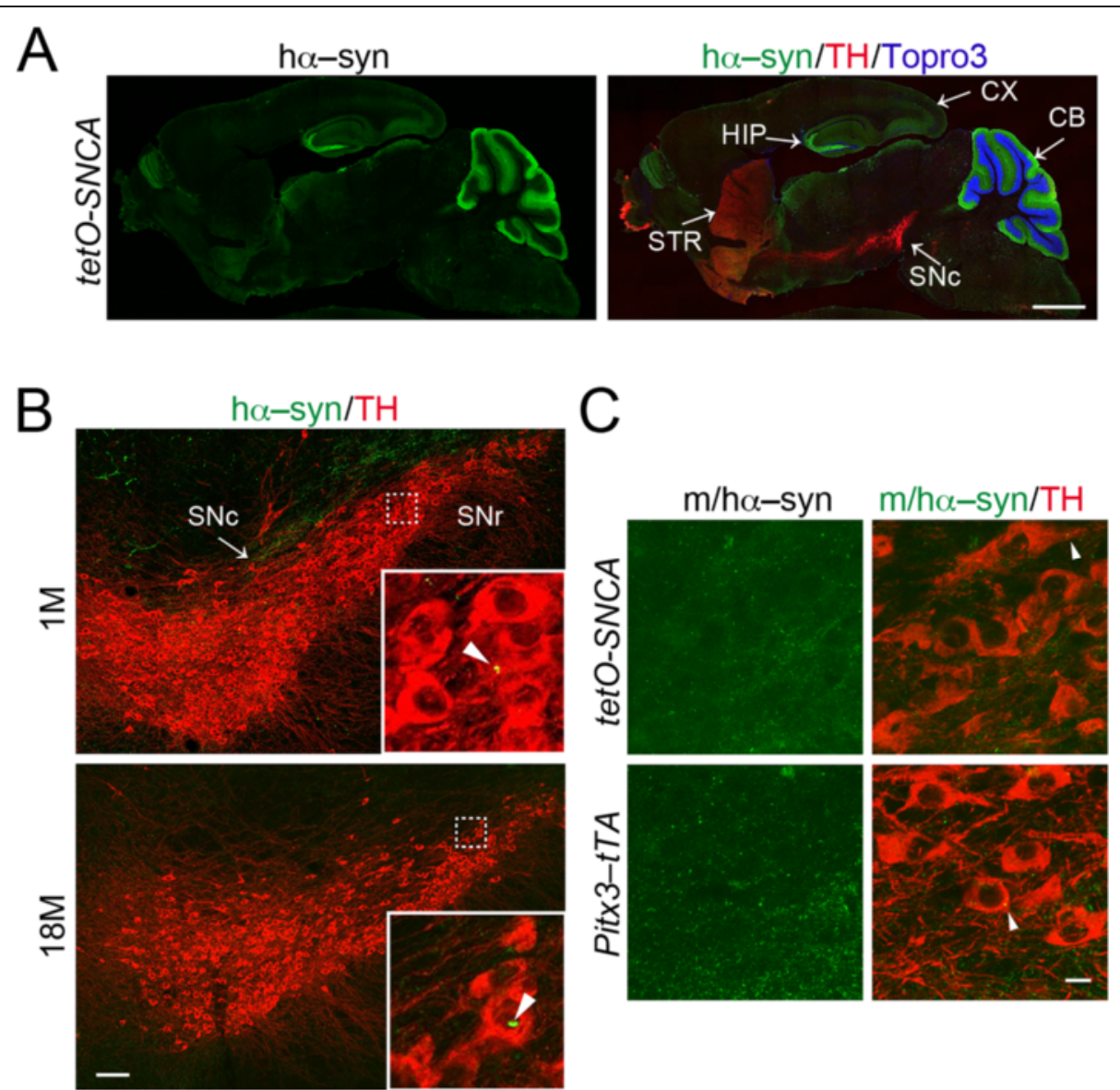

Fig. 1 No propagation of a-syn into the nigrostriatal DA neurons of tetO-SNCA single transgenic mice. a Sample images show the expression pattern of ha-syn (green) in the sagittal sections of 1-month-old tetO-SNCA mice. DA neurons were marked by TH staining (red). Topro3 was used for counter-staining (blue). CX; cerebral cortex; HIP: hippocampus; CB: cerebellum; STR: striatum; SNc: substantia nigra pars compacta. Scale bar: $1 \mathrm{~mm}$. b Sample images show the staining of ha-syn (green) and TH (red) in the SNc of 1- and 18-month-old tetO-SNCA mice. Insets highlight the boxed area. Arrowheads point to the ha-syn-positive puncta. SNr: substantia nigra pars reticulata. Scale bar: $100 \mu$ m. c Sample images show the staining of m/ha-syn (green) and TH (red) in the SNc of 18-month-old tetO-SNCA single transgenic and Pitx3-tTA heterozygous knock-in mice. Arrowheads point to the ha-syn-positive puncta. Scale bar: $10 \mu \mathrm{m}$

Thus, to test whether ho-syn would induce the aggregation of endogenous mouse $\alpha$-syn (m $\alpha-$ syn) in SNc DA neurons, we stained the midbrain sections of 18-month-old tetO-SNCA single transgenic and Pitx3$t T A$ heterozygous knock-in mice with an antibody that recognizes both mouse and human $\alpha-\operatorname{syn}(\mathrm{m} / \mathrm{h} \alpha-\mathrm{syn})$. We observed a similar number of small $\mathrm{m} / \mathrm{h} \alpha-$ syn-positive puncta in the SNc DA neurons of tetO-SNCA and control Pitx3-tTA mice (Fig. 1c), indicating a lack of recruitment of endogenous $\alpha$-syn. Together, these observations suggest a lack of long-range transneuronal propagation of transgenic h $\alpha-$ syn into SNc DA neurons during aging.

\section{a-synuclein is not transmitted from presynaptic terminals into SNC DA neurons}

We next examined the transmission between anatomically connected brain regions, specifically the SNc and striatum in the basal ganglia. The majority of DA neurons in the SNc send projections to SPNs in the striatum [19]. SPNs comprise two main subpopulations that form direct and indirect pathways in the basal ganglia [19]. In the direct pathway, most SPNs that express dopamine receptor D1 (Drd1) send projections to neurons at substantia nigra pars reticulata $(\mathrm{SNr})$, while some directly form synapses with SNc DA neurons [20, 21]. To further investigate propagation in the direct pathway, we used a line of mice that utilizes a reverse tetracycline transactivator (rtTA) and the Drd1a promoter, which directs transgene expression in the SPNs of direct pathway. When crossed with tetO-SNCA mice, we expect to see $\mathrm{h} \alpha-$ syn expression along the direct pathway. Indeed at 1 month-old, Drd1-rtTA/tetO-SNCA mice showed strong $\mathrm{h} \alpha-$ syn expression in the striatum and $\mathrm{SNr}$, but no expression in the SNc (Fig. 2a). Once again, h $\alpha-$ syn expression at later ages would indicate that propagation is 

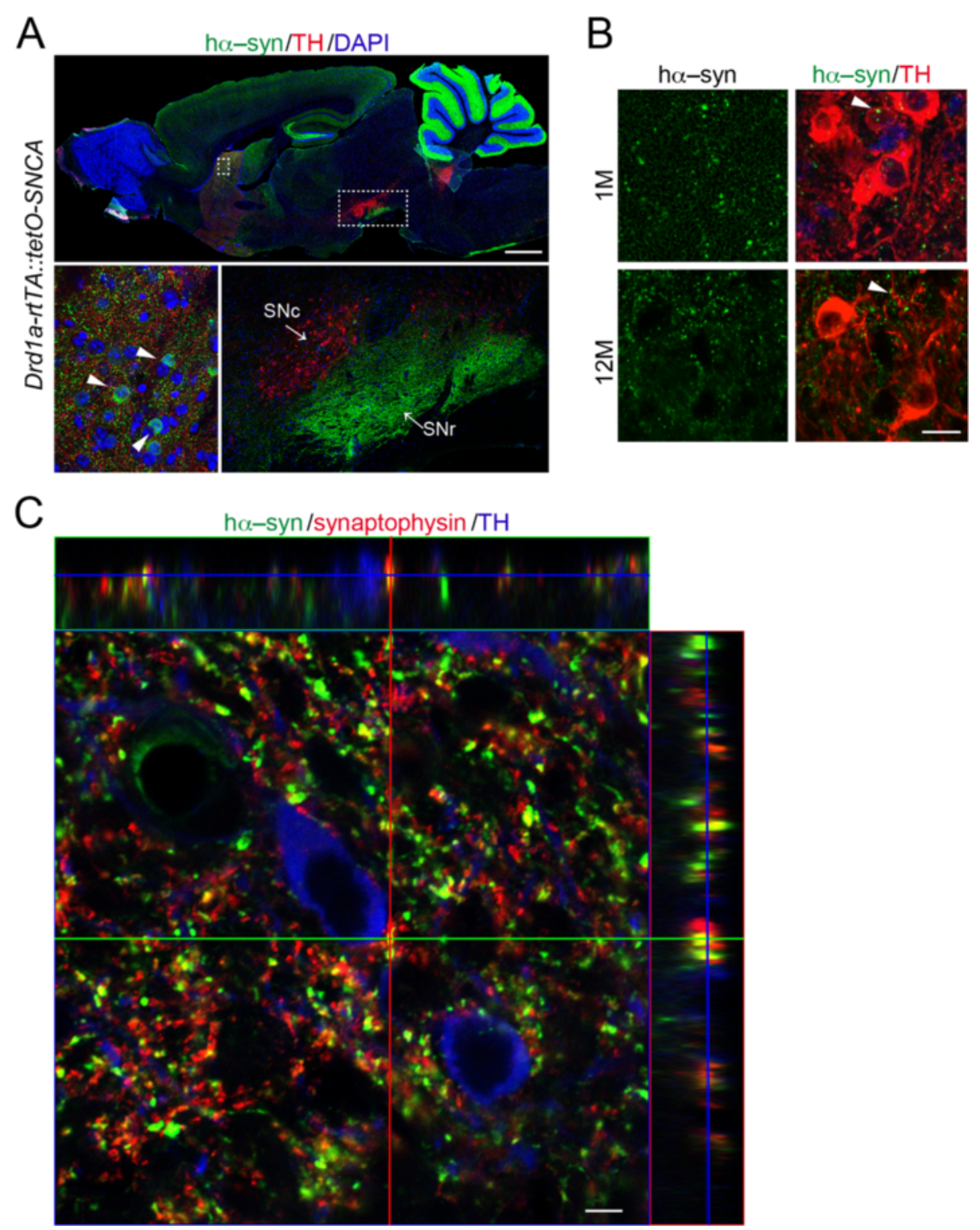

Fig. 2 a-synuclein is not transmitted in anatomically connected regions. a In the top panel, sample image shows the expression pattern of ha-syn (green) and TH (red) in the sagittal sections of 1-month-old Drd1a-rtTA.:tetO-SNCA bigenic mice. Topro3 was used for counter-staining (blue). In the bottom left panel, arrowheads point to Drd1-type striatal neurons that express ha-syn. The bottom right panel highlights the boxed area in the top panel. Scale bar: $1 \mathrm{~mm}$. b Sample images show the staining of ha-syn (green) and TH (red) in the SNc of 1- and 18-month-old Drd1a-rtTA.:tetO-SNCA bigenic mice. Arrowheads point to the ha-syn-positive puncta. Scale bar: $10 \mu \mathrm{m}$. c Sample image shows the staining of ha-syn (green), synaptophysin (red) and TH (blue) in the SNc of 18-month-old Drd1a-rtTA::tetO-SNCA bigenic transgenic mice. The panels at the top and right depict the distribution of different fluorophores along the $Y$ - and $X$-axis. Scale bar: $10 \mu m$

present. We then looked at 12-month-old mice and found that they too had no ho-syn-positive cells present in the $\mathrm{SNc}$ (Fig. 2b). As seen with the tetO-SNCA mice (Fig. 1b and c), small ho-syn-positive puncta were observed near or on top of SNc DA neurons (Fig. 2b). These puncta were also positive for synaptophysin, a marker for presynaptic terminals [22] (Fig. 2c), indicating a presynaptic enrichment of $\alpha$-syn as previously documented [23]. The same as the previous experiments, we again found no indication that ho-syn possesses the ability to propagate across the synapses.

\section{a-synuclein is unable to undergo cell-to-cell} transmission between SNc DA neurons

We finally examined $\alpha$-syn propagation within SNc DA neurons. To express SNCA in the midbrain, we crossbred Pitx3-tTA heterozygous knock-in mice with tetO-SNCA heterozygous transgenic mice to generate 
Pitx3-tTA::tetO-SNCA bigenic mice. The cells express-

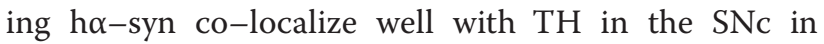
these mice (Fig. 3a). We subsequently examined colocalization patterns in the Pitx-tTA::tetO-SNCA bigenic mice that expressed h $\alpha$-syn in midbrain DA neurons and (Fig. 3b-d). We looked at mice that were 1 - and 16-18-month-old to determine how colocalization values changed with age. Pitx3 is mainly expressed in the ventral SNc DA neurons, but not in the dorsal ones that account for about $20 \%$ of total DA neuron population [24]. In our study, this translated to $\sim 80 \%$ of the TH-positive SNc cells expressing h $\alpha-$ syn under the control of the Pitx3 promoter (Fig. 3d). We wanted to see if this percentage would increase with age, indicating the presence of cell-to-cell transmission of ha-syn in these cells. Contrary to what would
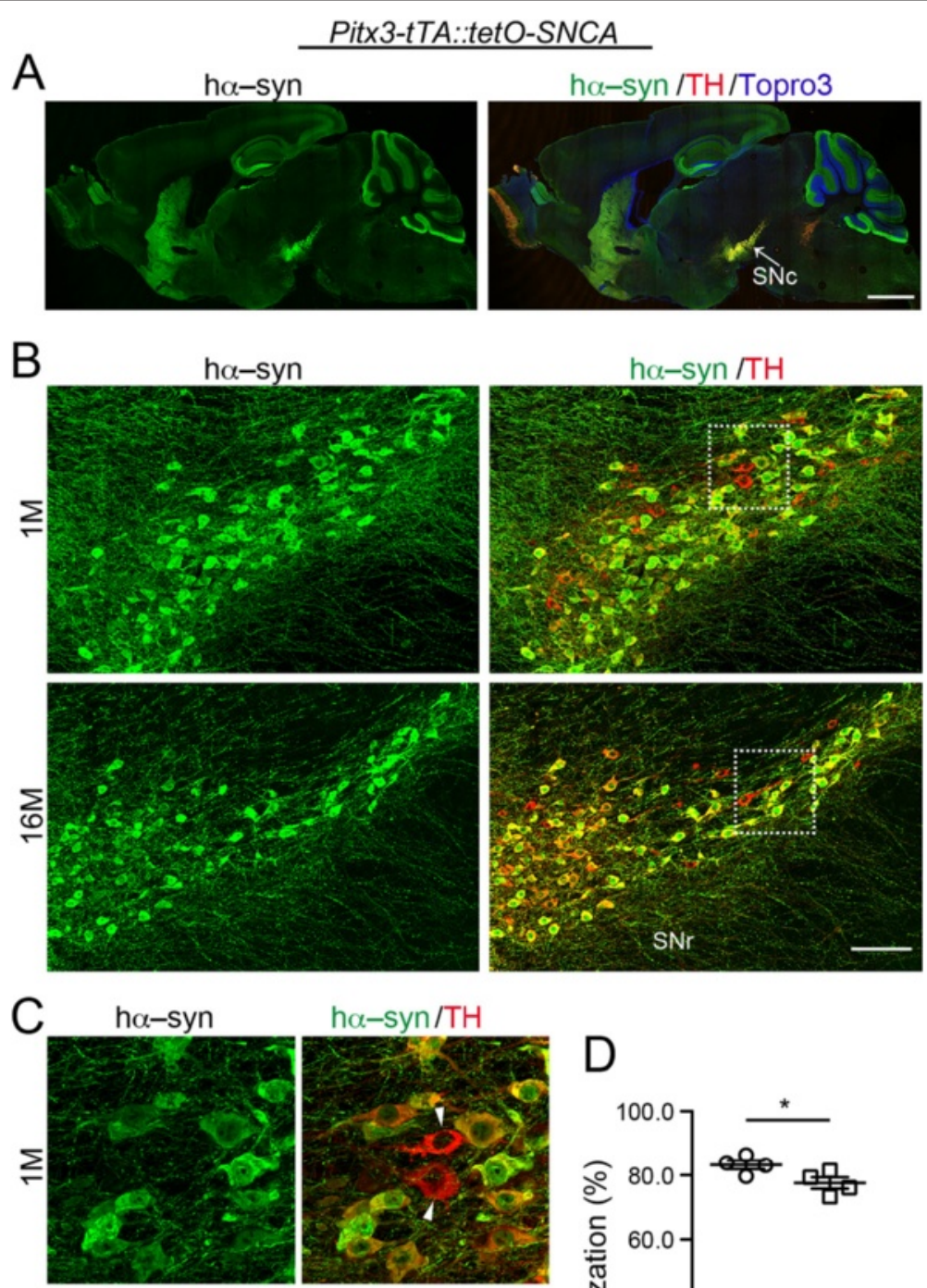

$\mathrm{h} \alpha-\mathrm{syn} / \mathrm{TH}$
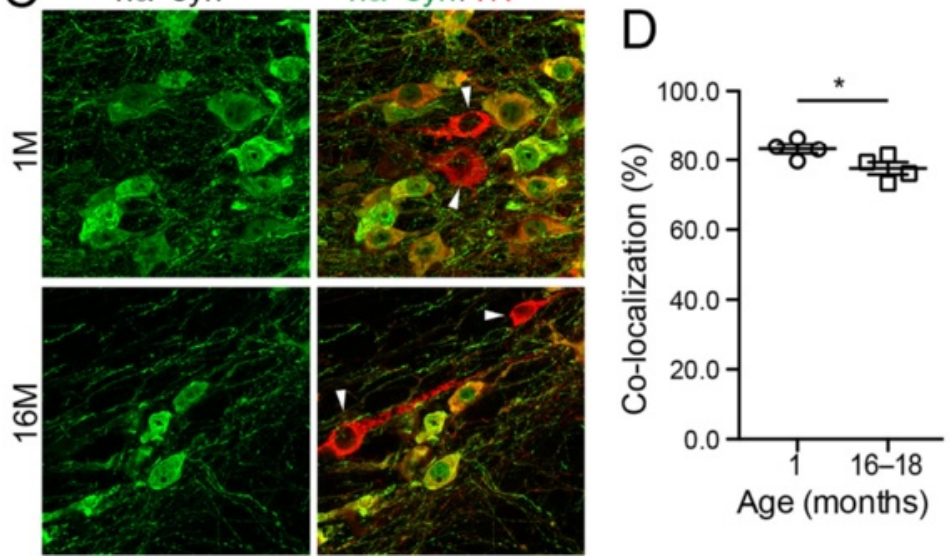

Fig. 3 a-syn is unable to undergo cell-to-cell transmission at SNc. a Sample images show the expression pattern of ha-syn (green) and TH (red) in the sagittal sections of 1-month-old Pitx3-tTA::tetO-SNCA bigenic mice. Topro3 was used for counter-staining (blue). Scale bar: $1 \mathrm{~mm}$. b Sample images show the staining of ha-syn (green) and TH (red) in the SNc of 1- and 16-month-old Pitx3-tTA.:tetO-SNCA bigenic mice. Scale bar: $100 \mu \mathrm{m}$. c Images highlight the boxed areas in $\mathbf{b}$. Arrowheads point to the ha-syn-negative DA neurons. d Scatter plot depicts co-localization percentages at 1 month and 16 months. Data were presented as mean \pm SEM. ${ }^{*} \mathrm{P}<0.05$ 
be expected for propagation, we found that the average percentage of co-localized cells at 1-month-old was $83.3 \%$, whereas the percentage at 16-18-month-old was $77.6 \%$ (Fig. 3d). As we did not see the increase that indicates the presence of propagation, this experiment provided no evidence for local propagation between neighboring SNc DA neurons.

In addition to tissue staining, Western blotting revealed more than 5 -fold increase of $\alpha$-syn expression in cerebellum of tetO-SNCA single and Pitx3-tTA::tetO-SNCA double transgenic mice (Fig. 4a). Furthermore, $\alpha$-syn-positive high molecular weight (HMW) bands were also detected in the whole brain homogenates of tetO-SNCA single, Pitx3-tTA::tetO-SNCA, and Drd1-rtTA::tetO-SNCA double transgenic mice
(Fig. 4b), suggesting the existence of $\alpha$-syn aggregates in the mouse brains of all the transgenic lines. Western bolting also showed the h $\alpha$-syn expression was substantially increased in the striatum of Pitx3-tTA::tetO-SNCA double transgenic mice compared to the tetO-SNCA single animals (Fig. 4c), resulting from the projection of ho-syn-expressing DA axons at the striatum (Fig. 3a).

\section{Discussion}

We show here that transgenic h $\alpha$-syn does not show detectable propagation to nigrostriatal DA neurons in various mouse models. We first used tetO-SNCA single transgenic mice to show that we could not observe long-range propagation of ha-syn into SNc DA

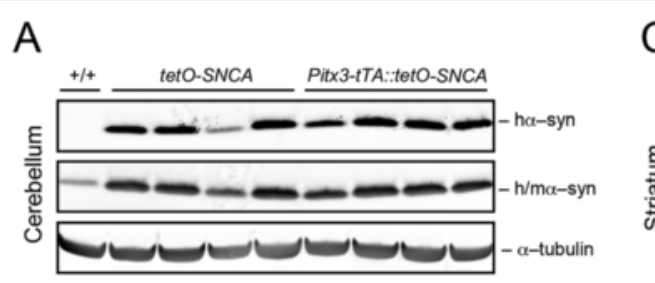

C

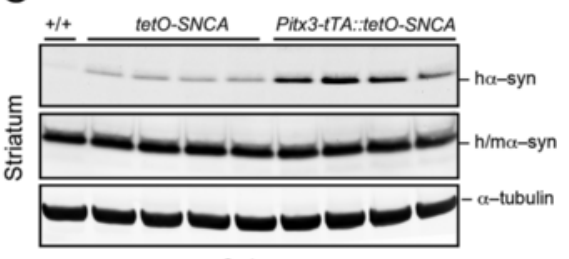

Cerebellum
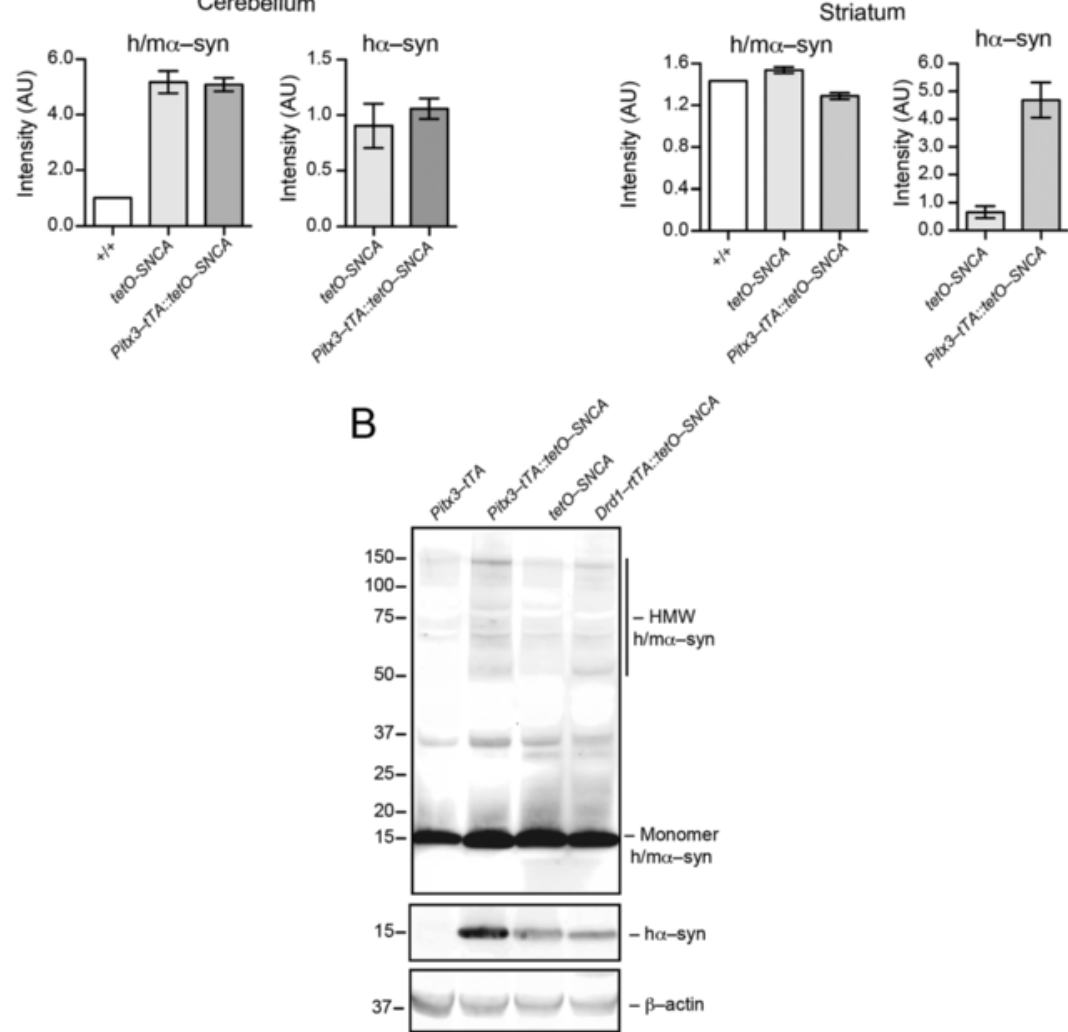

Fig. 4 Overexpression of ha-syn in tetO-SNCA single and Pitx3-tTA.:tetO-SNCA bigenic mice. a Western blots show expression of ha-syn and $\mathrm{m} /$ ha-syn in the cerebellum of 1-month-old tetO-SNCA single and Pitx3-tTA.:tetO-SNCA bigenic mice. a-tubulin was used as loading control. Bar graphs depict the signal intensity. Data were presented as mean \pm SEM. $\mathbf{b}$ Western blot shows expression of ha-syn and $m / h a-s y n$ in the whole brain of 18-month-old Pitx3-tTA, tetO-SNCA single, Drd1a-rtTA::tetO-SNCA bigenic and Pitx3-tTA:tetO-SNCA bigenic mice. $\beta$-actin was used as loading control. c Western blots show expression of ha-syn and m/ha-syn in the striatum of 1-month-old tetO-SNCA single and Pitx3-tTA.:tetO-SNCA bigenic mice. a-tubulin was used as loading control. Bar graphs depict the signal intensity. Data were presented as mean \pm SEM 
neurons. These mice have no transgenic h $\alpha-$ syn expression in the nigrostriatal pathway; however, they do have "leaky", non-specific h $\alpha$-syn expression in other brain regions (i.e. hippocampus, cortex, and cerebellum). Any of these other regions could have played a role being a source of $\alpha$-syn if the protein could indeed propagate. We performed immunohistochemical experiments on young and aged mice to see if we can observe h $\alpha$-syn-positive staining anywhere in the SNc DA neurons of aged animals. However, these experiments gave no indication that long-range propagation was present in SNCA mice.

The following experiment tested propagation that may occur through neuronal projections from neighboring brain regions. For these experiments, we utilized a line of mice, Drd1a-rtTA::tetO-SNCA, which had ho-syn expression in the striatum and $\mathrm{SNr}$, modeling the direct pathway of the basal ganglia. At 1-month of age, these mice exhibited no ho-syn expression in the SNc DA neurons. If propagation was present, we should be able to see ho-syn expression at later ages in these mice. This may have occurred as transmission directly to the SNc from the SPNs that form synapses onto SNc DA neurons [21]. However, as with the previous experiments, we found no evidence of h $\alpha$-syn being present in the SNc, again showing that there was no evident propagation.

Finally, we looked at Pitx3-tTA::tetO-SNCA bigenic mice, which utilize the Pitx3 driver to promote h $\alpha$-syn expression along the nigrostriatal pathway, in addition to the leaky expression patterns seen in the tetO-SNCA single transgenic mice. Following the expression pattern of Pitx3, we found that $\sim 80 \%$ of cells were both h $\alpha$-syn-positive and TH-positive in 1-month-old bigenic mice. An increase in this percentage in aged mice would indicate that more cells were becoming h $\alpha$-syn-positive, thus giving evidence for cell-cell transmission of $\alpha$-syn. While there was about $50 \%$ loss of SNc DA neurons in Pitx3-tTA::tetO-SNCA bigenic mice compared to Pitx3-tTA knock-in mice, no further degeneration occurred between 1-month and 16-18-month-old bigenic mice (Fig. 5a-b). Thus any increase could be attributed to the spread of $\alpha-$ syn, as opposed to cell death that may have resulted from $\alpha-$ syn toxicity. Instead of seeing the increase that would indicate propagation, we actually saw a slight decrease. However, the lack of degeneration led us to conclude that this decrease likely has no actual significance in the pathogenesis.

\section{Conclusion}

Many studies have shown the ability of $\alpha$-syn to propagate with the use of stereotaxic injections of preformed fibrils and have provided very convincing data for the ability of $\alpha$-syn to behave as a prion, both in neurons and in glial cells [14]. However, these studies often take advantage of artificial injections or inoculations, which may not be as applicable in a clinical, physiological setting. Therefore, alternative explanations to the prion hypothesis cannot be dismissed, including oxidative stress, excitotoxicity, neuroinflammation, and loss of neurotrophic factor support.

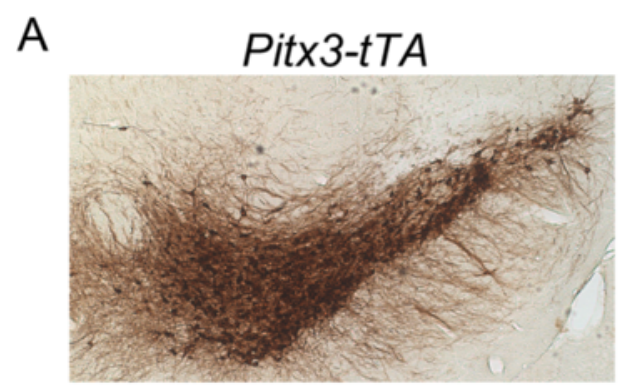

Pitx3-tTA::tetO-SNCA

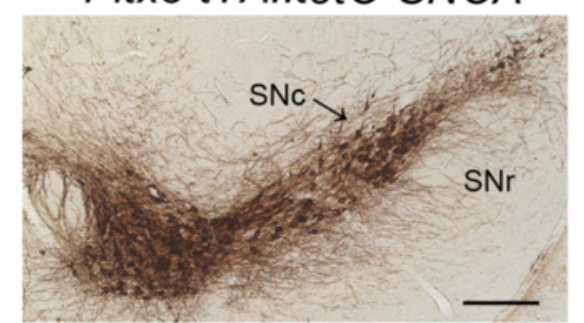

B

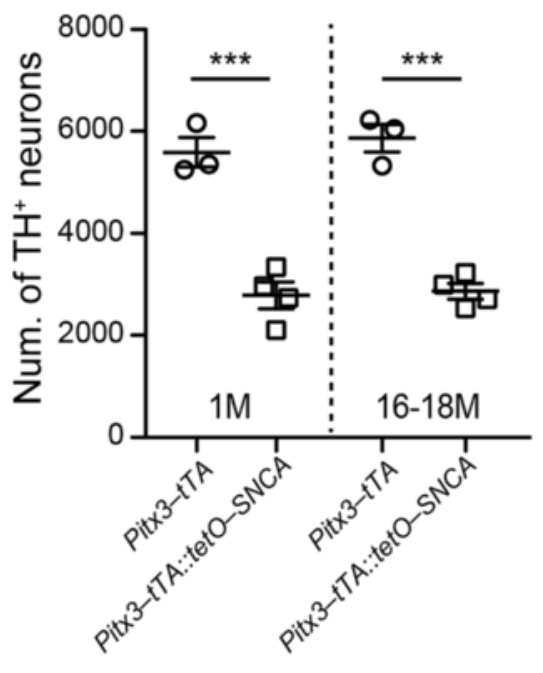

Fig. 5 Loss of DA neurons in the SNc of Pitx3-tTA.:tetO-SNCA bigenic mice. a Sample images show the staining of TH (brown) in the SNc of 16-month-old Pitx3-tTA heterozygous knock-in and Pitx3-tTA::tetO-SNCA bigenic mice. Scale bar: $100 \mu \mathrm{m}$. b Scatter plot depicts the number of remaining $\mathrm{TH}$-positive neurons in the SNc of 1 month and 16-18 months. Data were presented as mean $\pm \mathrm{SEM}$. ${ }^{* * *} \mathrm{P}<0.001$ 
These alternative explanations are not mutually exclusive and may potentially induce pathogenesis in a synergistic manner. Future studies should focus on microglial activation and other inflammatory responses in the brain resulting from intracerebral injections and inoculations. In addition, further scrutiny into the effect of inflammation on $\alpha$-syn expression can provide answers about the causes and mechanisms by which $\alpha$-syn adopts its abnormal prion-like qualities.

\section{Competing interests}

The authors declare that they have no competing interests.

\section{Authors' contributions}

NS carried out immunostaining experiments and data analyses, drafted the manuscript. ZW provided additional immunostaining data. GL, CS, LS, and CX provided tetO-SNCA transgenic mice and initial characterization. HW, XC, MC, and PC performed Western blot. LS provided mice. HC designed the experiments and wrote the manuscript. All authors read and approved the final manuscript.

\section{Acknowledgement}

This work was supported by the intramural research program of National Institute on Aging (HC: AG-000928, 929). The authors would like to thank members of Cai lab for providing various supports.

\section{Author details \\ ${ }^{1}$ Transgenics Section, Laboratory of Neurogenetics, National Institute on Aging, National Institutes of Health, Building 35, Room 1A112, MSC 3707, 35 Convent Drive, Bethesda, MD 20892-3707, USA. ${ }^{2}$ Unit on Synapse Development Plasticity, Clinical Brain Disorder Branch, National Institute of Mental Health, National Institutes of Health, Bethesda, MD 20892, USA. ${ }^{3}$ Present addresses: Feinberg School of Medicine, Northwestern University, Chicago, IL 60611, USA. ${ }^{4}$ Present addresses: Swarthmore College, Swarthmore, PA 19081, USA. ${ }^{5}$ Present addresses: Centennial High School, Elicott City, MD 21042, USA. ${ }^{6}$ Present addresses: George Washington University, Washington, DC 20052, USA.}

Received: 7 October 2015 Accepted: 1 December 2015

Published online: 03 December 2015

\section{References}

1. Thomas B, Beal MF. Parkinson's disease. Hum Mol Genet. 2007;16(Spec No. 2):R183-94. doi:10.1093/hmg/ddm159.

2. Tolosa $E$, Pont-Sunyer $C$. Progress in defining the premotor phase of Parkinson's disease. J Neurol Sci. 2011;310(1-2):4-8. doi:10.1016/j.jns. 2011.05.027.

3. Spillantini MG, Schmidt ML, Lee VM, Trojanowski JQ, Jakes R, Goedert M. Alpha-synuclein in Lewy bodies. Nature. 1997;388(6645):839-40. doi: 10.1038/42166.

4. Picconi B, Piccoli G, Calabresi P. Synaptic dysfunction in Parkinson's disease. Adv Exp Med Biol. 2012;970:553-72. doi:10.1007/978-3-7091-0932-8_24.

5. Hardy J, Cai H, Cookson MR, Gwinn-Hardy K, Singleton A. Genetics of Parkinson's disease and parkinsonism. Ann Neurol. 2006;60(4):389-98. doi:10. 1002/ana.21022.

6. Braak H, Del Tredici K, Rub U, de Vos RA, Jansen Steur EN, Braak E. Staging of brain pathology related to sporadic Parkinson's disease. Neurobiol Aging. 2003;24(2):197-211.

7. Volpicelli-Daley LA, Luk KC, Patel TP, Tanik SA, Riddle DM, Stieber A, et al. Exogenous alpha-synuclein fibrils induce Lewy body pathology leading to synaptic dysfunction and neuron death. Neuron. 2011;72(1):57-71. doi:10. 1016/.j.neuron.2011.08.033.

8. Aulic S, Le T, Moda F, Abounit S, Corvaglia S, Casalis L, et al. Defined alpha-synuclein prion-like molecular assemblies spreading in cell culture. BMC Neurosci. 2014;15:69. doi:10.1186/1471-2202-15-69.

9. Luk KC, Kehm V, Carroll J, Zhang B, O'Brien P, Trojanowski JQ, et al. Pathological alpha-synuclein transmission initiates Parkinson-like neurodegeneration in nontransgenic mice. Science. 2012;338(6109):949-53. doi:10.1126/science.1227157.
10. Luk KC, Kehm VM, Zhang B, O'Brien P, Trojanowski JQ, Lee VM. Intracerebral inoculation of pathological alpha-synuclein initiates a rapidly progressive neurodegenerative alpha-synucleinopathy in mice. J Exp Med. 2012;209(5): 975-86. doi:10.1084/jem.20112457.

11. Betemps D, Verchere J, Brot S, Morignat E, Bousset L, Gaillard D, et al. Alpha-synuclein spreading in M83 mice brain revealed by detection of pathological alpha-synuclein by enhanced ELISA. Acta Neuropathol Commun. 2014;2:29. doi:10.1186/2051-5960-2-29.

12. Watts JC, Giles K, Oehler A, Middleton L, Dexter DT, Gentleman SM, et al. Transmission of multiple system atrophy prions to transgenic mice. Proc Natl Acad Sci U S A. 2013;110(48):19555-60. doi:10.1073/pnas.1318268110.

13. Recasens A, Dehay B, Bove J, Carballo-Carbajal I, Dovero S, Perez-Villalba A, et al. Lewy body extracts from Parkinson disease brains trigger alpha-synuclein pathology and neurodegeneration in mice and monkeys. Ann Neurol. 2014;75(3):351-62. doi:10.1002/ana.24066.

14. Luk KC, Lee VM. Modeling Lewy pathology propagation in Parkinson's disease. Parkinsonism Relat Disord. 2014;20 Suppl 1:585-7. doi:10.1016 S1353-8020(13)70022-1.

15. Jankowsky UL, Savonenko A, Schilling G, Wang J, Xu G, Borchelt DR. Transgenic mouse models of neurodegenerative disease: opportunities for therapeutic development. Curr Neurol Neurosci Rep. 2002;2(5):457-64.

16. Lin X, Parisiadou L, Sgobio C, Liu G, Yu J, Sun L, et al. Conditional expression of Parkinson's disease-related mutant alpha-synuclein in the midbrain dopaminergic neurons causes progressive neurodegeneration and degradation of transcription factor nuclear receptor related 1. J Neurosci. 2012;32(27):9248-64. doi:10.1523/JNEUROSCI.1731-12.2012.

17. Gossen M, Bujard H. Tight control of gene expression in mammalian cells by tetracycline-responsive promoters. Proc Natl Acad Sci U S A. 1992;89(12): 5547-51.

18. Smidt MP, Smits SM, Bouwmeester H, Hamers FP, van der Linden AJ, Hellemons AJ, et al. Early developmental failure of substantia nigra dopamine neurons in mice lacking the homeodomain gene Pitx3. Development. 2004;131(5):1145-55. doi:10.1242/dev.01022.

19. Gerfen CR, Surmeier DJ. Modulation of striatal projection systems by dopamine. Annu Rev Neurosci. 2011;34:441-66. doi:10.1146/annurevneuro-061010-113641.

20. Gerfen CR. The neostriatal mosaic: compartmentalization of corticostriatal input and striatonigral output systems. Nature. 1984;311(5985):461-4.

21. Watabe-Uchida M, Zhu L, Ogawa SK, Vamanrao A, Uchida N. Whole-brain mapping of direct inputs to midbrain dopamine neurons. Neuron. 2012; 74(5):858-73. doi:10.1016/j.neuron.2012.03.017.

22. Wiedenmann B, Franke WW. Identification and localization of synaptophysin, an integral membrane glycoprotein of $\mathrm{Mr} 38,000$ characteristic of presynaptic vesicles. Cell. 1985;41(3):1017-28.

23. Maroteaux L, Campanelli JT, Scheller RH. Synuclein: a neuron-specific protein localized to the nucleus and presynaptic nerve terminal. J Neurosci. 1988;8(8):2804-15.

24. Bifsha P, Yang J, Fisher RA, Drouin J. Rgs6 is required for adult maintenance of dopaminergic neurons in the ventral substantia nigra. PLoS Genet. 2014; 10(12):e1004863. doi:10.1371/journal.pgen.1004863.

\footnotetext{
Submit your next manuscript to BioMed Central and we will help you at every step:

- We accept pre-submission inquiries

- Our selector tool helps you to find the most relevant journal

- We provide round the clock customer support

- Convenient online submission

- Thorough peer review

- Inclusion in PubMed and all major indexing services

- Maximum visibility for your research
}

Submit your manuscript at www.biomedcentral.com/submit

(O) BioMed Central 